Supporting Information for

\title{
Revealing Conformational Transition Dynamics of Photosynthetic Proteins in Single-Molecule Electrical Circuits
}

Zhiheng Yang, ${ }^{\dagger}$ Chenhui $\mathrm{Qi},{ }^{\dagger}$ Wenzhe Liu, Dongbao Yin, Longjiang Yu, ${ }^{*}$ Lidong $\mathrm{Li}^{*}$ and Xuefeng Guo*

[*]Zhiheng Yang ${ }^{\dagger}$, Dongbao Yin, Prof. Lidong Li

State Key Laboratory for Advanced Metals and Materials, School of Materials Science and Engineering, University of Science and Technology Beijing, Beijing 100083, P. R. China.

Email: lidong@mater.ustb.edu.cn

Wenzhe Liu, Prof. Xuefeng Guo

Beijing National Laboratory for Molecular Sciences State Key Laboratory for Structural Chemistry of Unstable and Stable Species, College of Chemistry and Molecular Engineering, Peking University Beijing 100871, P. R. China.

Email: guoxf@pku.edu.cn

Chenhui Qi, ${ }^{\dagger}$ Prof. Longjiang Yu

Photosynthesis Research Center, Key Laboratory of Photobiology, Institute of Botany, Chinese Academy of Sciences, Beijing 100093, P. R. China. Email: longer@ibcas.ac.cn

†These authors contributed equally to this work. 


\section{Table of Contents}

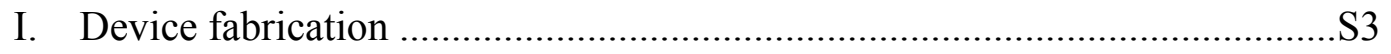

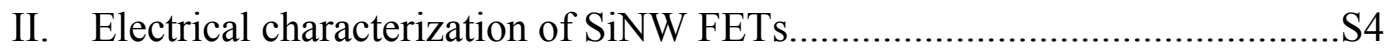



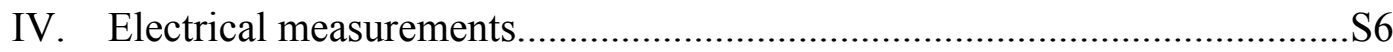

V. Dynamics analysis of the LH1-RC vibration..............................................S10

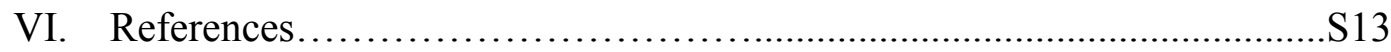




\section{Device fabrication}

The silicon nanowire growth procedure is similar to those reported in the previous studies. ${ }^{1,2}$ Silicon wafers with a $1000 \mathrm{~nm}$-thick thermal oxide layer were used as growth substrates and gold nanoparticles with an average diameter of $20 \mathrm{~nm}$ (Ted Pella) were used as catalysts. Boron-doped $p$-type SiNWs were synthesized at $465{ }^{\circ} \mathrm{C}$ for about 25 min, by using $2.5 \mathrm{sccm}$ disilane (Matheson Gas Products, 99.998\% Purity) as the reactant source, $0.11 \mathrm{sccm}$ diborane (100 ppm, diluted in $\left.\mathrm{H}_{2}\right)$ as the $p$-type dopant with a $\mathrm{B} / \mathrm{Si}$ ratio of $1 / 100000$ and $7.5 \mathrm{sccm} \mathrm{H}_{2}$ as the carrier gas.
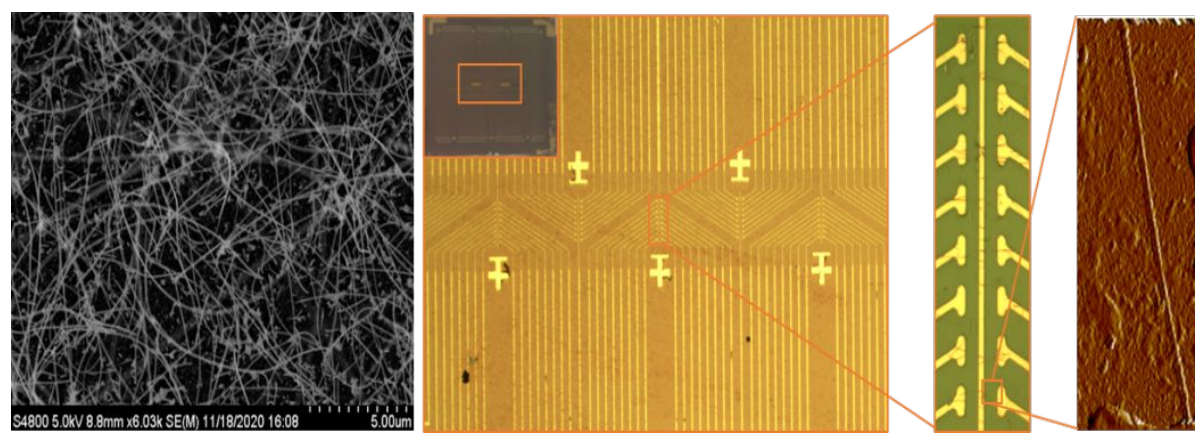

Figure S1. Optical and SEM images of a high-density SiNW FET array. Left inset shows a pattern we designed with 96 pairs of electrodes to make transistor arrays.

Based on a mechano-sliding method, SiNWs were transferred in the assigned region designed for electrodes patterning on the surface of a doped silicon wafer with circa $1000 \mathrm{~nm}$. After a positive resist (AR-P 5350) window was formed by photolithography to retain the SiNWs between the electrodes and remove the redundant SiNWs with the aid of sonication, the resist was washed by acetone in the end. Then, to form better Ohmic electrical contacts with metal electrodes, SiNWs were immersed in the buffered HF solution $\left(40 \% \mathrm{NH}_{4} \mathrm{~F}: 40 \% \mathrm{HF}, 7: 1\right)$ to remove the oxide shell of the nanowires. After that, $8 \mathrm{~nm} \mathrm{Cr}$ and sequent $80 \mathrm{~nm}$ Au were deposited by utilizing thermal evaporation (ZHD-300, Beijing Technol Science) to emerge metal electrodes. In order to passivate the contact interface, another $50 \mathrm{~nm}$ thick $\mathrm{SiO}_{2}$ protective layer was deposited through electron beam thermal evaporation (TEMD-600, Beijing Technol Science). Next, photoresist among the electrodes was washed by copious acetone to obtain SiNW FET arrays. Given the fact that the drain current is immersed in the solution, a negative resist (SU-8, 2002) window was opened by photolithography to protect the majority of the surface finally. By adopting this method, we were able to prepare high-density transistor arrays (Figures S1 and S2). 


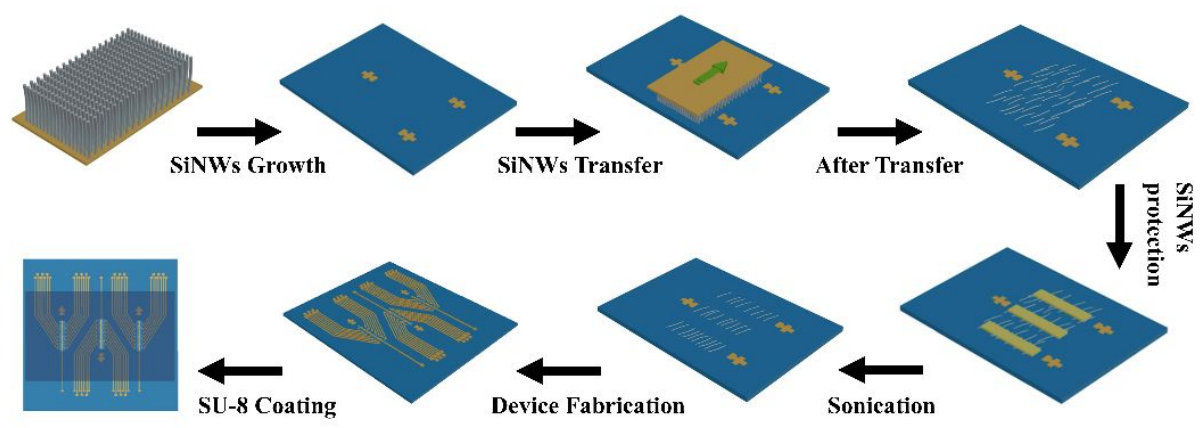

Figure S2. The brief process of mechano-sliding transfer of SiNWs and FET array fabrication.

\section{Electrical characterization of SiNW FETs}

After SiNW transistor fabrication, the electrical characterizations of these transistors were carried out at room temperature in the ambient by using an Agilent 4155C semiconductor analyzer and a Karl Süss (PM5) manual probe station. Heavily doped Si substrates was used as the global back gate. Figure S3 demonstrates that SiNW transistors show the typical $p$-type behaviors with good Ohmic contacts. ${ }^{3}$

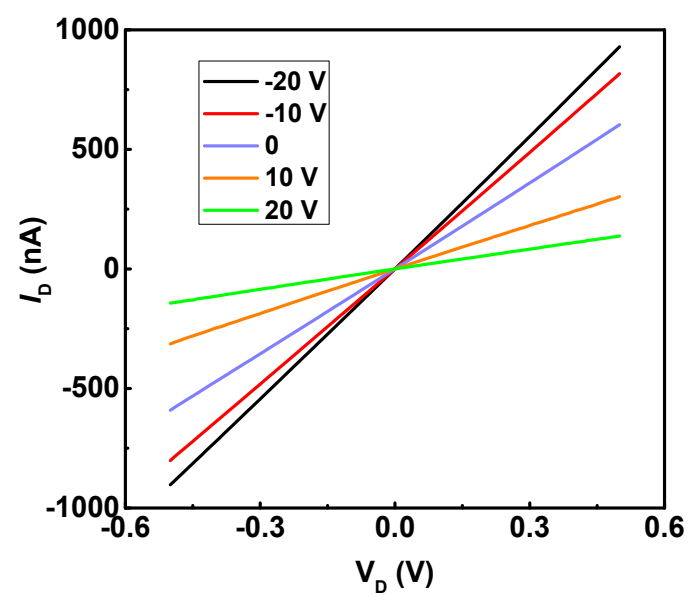

Figure S3. Output characteristics for a $p$-type SiNW FET device when $V_{\mathrm{D}}$ scans from $0.5 \mathrm{~V}$ to $-0.5 \mathrm{~V}$ at different gate voltages $(-20,-10,0,10$ and $20 \mathrm{~V})$. 


\section{Single LH1-RC protein decoration}

gap opening. The high-quality SiNW FET device was coated by a PMMA layer (950, A4) through spinner and baked at $180^{\circ} \mathrm{C}$ for $2 \mathrm{~min}$. Utilizing high-resolution electron beam lithography with a designed CAD file which includes a $\sim 10 \mathrm{~nm}$-wide line at the potential position generated the window precursor. Then, with the aid of sonication, the resist was developed in a mixture of water/isopropanol $(\mathrm{V}: \mathrm{V}=1: 3)$ for the lift-off at $4{ }^{\circ} \mathrm{C}$ for $1 \mathrm{~min}$. After that, the devices were washed by deionized water and dried with $\mathrm{N}_{2}$ gas-stream. In order to completely remove the amorphous $\mathrm{SiO}_{2}$ layer and expose the $\mathrm{Si}-\mathrm{H}$ bond in the window area, the devices were immersed into the HF solution buffered with $\mathrm{NH}_{4} \mathrm{~F}\left(\mathrm{HF}(40 \%): \mathrm{NH}_{4} \mathrm{~F}(40 \%)=1: 7\right)$ for $7 \mathrm{~s}$, according to the etching rate we figured out in a previous report, ${ }^{2}$ and PMMA layer was peeled by acetone eventually. Applying sophisticated electron beam lithography and precise HF wet etching, a nanoscale window on the side of SiNWs was obtained to confine a single biomolecule.

Single LH1-RC protein biodecoration. The subsequent step is to achieve the attachment of biomolecules at the single-molecule level, where the key is the hydrosilylation of Si-H bonds. In order to improve the yield of alkyne hydrosilylation, we used undecynic acid as the grafted reagent and obtained carboxyl-terminated functionalization via heat reaction. ${ }^{2}$ The freshly-etched devices and 3-mg powders of undecynic acid were placed into a Schlenk bottle. After reaction under argon atmosphere at $90^{\circ} \mathrm{C}$ for $10 \mathrm{~h}$, the devices were soaked in dichloromethane and sonicated for $30 \mathrm{~s}$ to remove unreacted residues on the surface, and dried with a stream of $\mathrm{N}_{2}$ gas. After grafting, the devices were immersed in an aqueous mixed solution of $\mathrm{N}$ hydroxysuccinimide (NHS) (20 $\mathrm{mM})$ and 1-ethyl-3-(3- dimethylaminopropyl) carbodiimide (EDC) $(10 \mathrm{mM})$ and allowed to react at room temperature for $2 \mathrm{~h}$ so that carboxyl can be esterified by oxhydryl of NHS $(\mathrm{pH}=6.5)$. The devices were washed thoroughly with deionized water and then dried under a stream of $\mathrm{N}_{2}$ gas. The detailed characterizations have been shown in our previous report. Then, the devices were soaked in a N, N-Dimethylformamide (DMF) solution including N-(2-Aminoethyl) maleimide Hydrochloride $(18 \mathrm{mM})$ for $8 \mathrm{~h}$, so that the activated carboxyl was reacted with maleimide efficiently. Afterward, the devices were washed thoroughly with DMF and then dried under a stream of $\mathrm{N}_{2}$ gas. Eventually, the surface of the devices was covered by a $50 \mu \mathrm{L}$ LH1-RC solution ( $4.8 \mu \mathrm{M}$ LH1-RC complex in $10 \mathrm{mM}$ PBS (20 
$\mathrm{mM}$ Tricine, $5 \mathrm{mM} \mathrm{MgCl} 2,5 \mathrm{mM} \mathrm{KCl}$ and $0.03 \%$ n-Dodecyl- $\beta$-D-Maltopyranoside $(\mathrm{DDM}), \mathrm{pH}=7.8)$ ) for $24 \mathrm{~h}$ at $4{ }^{\circ} \mathrm{C}$. Before used for electrical measurements, the devices were washed by the buffer solution $(10 \mathrm{mM}, \mathrm{pH}=7.8)$ for $2 \mathrm{~min}$ (Figure S4).

\section{Optical characterization.}

The decorated device was covered by a buffer liquid layer and then attached with a coverslip. The fluorescence was excited by a $405 \mathrm{~nm}$ laser and monitored by the EMCCD through an 100x objective lens. 5000 photos taken by a super-resolution fluorescence microscopy with a 50-ms exposure were used to reconstruct through Stochastic Optical Reconstruction Microscopy (STORM), producing a single-protein resolution photograph and an enlarged image without the background. ${ }^{4}$
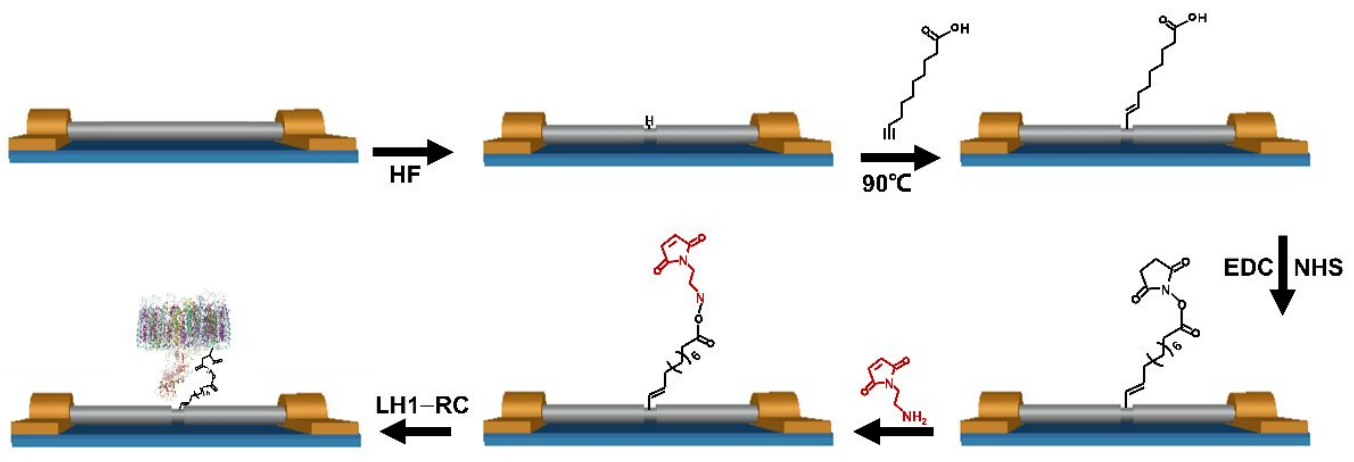

Figure S4. Schematic demonstration of the strategy used for surface functionalization and LH1-RC protein decoration.

\section{Electrical measurements}

The LH1-RC decorated devices were covered by a PDMS chunk with a hole of approximately $2 \mathrm{~mm}$ diameter acting as a reaction chamber on the surface of the device for electrical measurements and infused a $50-\mu \mathrm{L}$ PBS solution in the chamber. Then, the devices were fixed on the testing platform, which was assembled by a manual probe station and a hot/cold chuck (INSTEC, mK2000) with a proportion-integrationdifferentiation (PID) control system and liquid nitrogen cooling system capable of precisely controlling the temperatures. The source-drain and gate bias were always held at $V_{\mathrm{D}}=300 \mathrm{mV}$ and $V_{\mathrm{G}}=0 \mathrm{mV}$ by a HF2LI lock-in amplifier respectively (Zurich Instruments) in all real-time electrical measurements. The real-time current was amplified by a DL1211 preamplifier operating at $10^{7} \mathrm{~V} / \mathrm{A}$ gain and measurement data 
were collected by the HF2LI lock-in amplifier at $57.6 \mathrm{kHz}$ bandwidth and $57.6 \mathrm{KSa} / \mathrm{s}$ sampling rate, achieving a microsecond timescale resolution.

Before the decoration of a single LH1-RC protein, the control SiNW device which grafted with the molecular bridge was measured under pure buffer at different temperatures. The current data demonstrated that the device showed a one-level steady conductance (Figure S5). However, after the decoration of a single LH1-RC protein, the SiNW Device exhibited four current values and the measurements data can be idealized by a QUB software into four conducting states, as shown in Figure 1c. Furthermore, Figure 2 demonstrates a set of current data measured at five different temperatures varying from $15^{\circ} \mathrm{C}$ to $55^{\circ} \mathrm{C}$ with a $10{ }^{\circ} \mathrm{C}$ warming interval under light illumination and in the dark at each temperature. In order to verify the liability and efficiency, we repeated the measurement at other devices under the same condition. The reproducible results were shown in Figure S6 and Figure S7.


Figure S5. Electrical measurement of the control SiNW Device in the dark (left) and light illumination (right). 

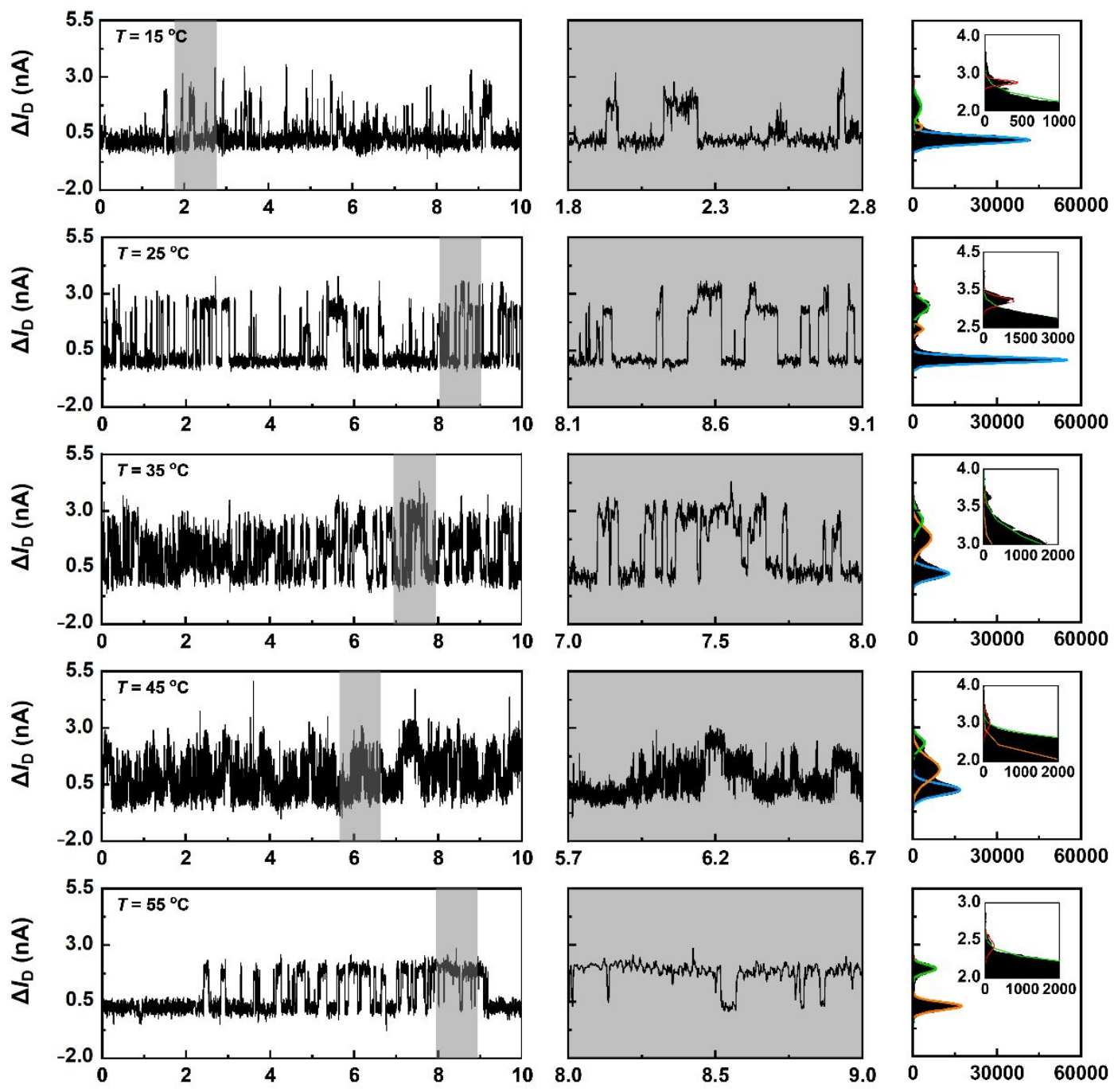

Figure S6. Temperature dependence of the LH1-RC complex vibration in the dark of another device. These graphs show time-averaged data sets of a representative LH1-RC complex vibration measured in a PBS buffer solution in the dark at five different temperatures varying from $15^{\circ} \mathrm{C}$ to $55^{\circ} \mathrm{C}$. The left graphs are real-time current recordings for a duration of $12 \mathrm{~s}$. The middle graphs are amplified 1-s data. The right graphs are the corresponding current histograms. 

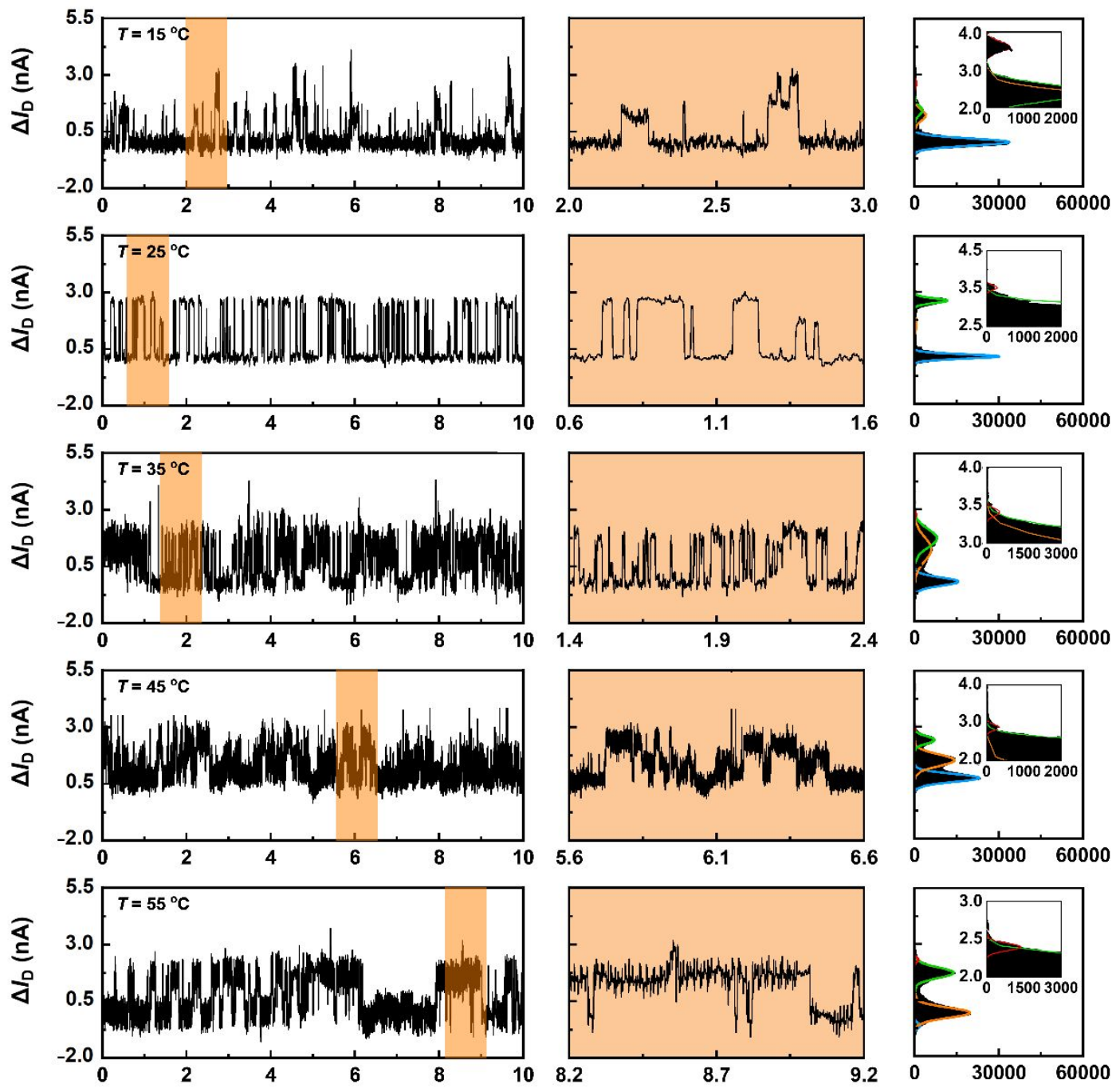

Figure S7. Temperature dependence of the LH1-RC complex vibration under illumination of another device. These graphs show time-averaged data sets of representative LH1-RC complex vibration measured in a PBS buffer solution under light illumination at five different temperatures varying from $15^{\circ} \mathrm{C}$ to $55^{\circ} \mathrm{C}$. The left graphs are real-time current recordings for a duration of $12 \mathrm{~s}$. The middle graphs are amplified 1-s data. The right graphs are the corresponding current histograms. 


\section{Dynamics analysis of the LH1-RC vibration}

In consideration of the signal and event similarity between single-molecule FRET and SiNW FET, we applied a QUB software in analyzing the data, which is extensively used for analyzing single-molecule fluorescent signals. We used the QUB software to idealize the data to a four-level fluctuation and then extracted the dwell times of four states. As depicted in Figure S8 and Figure S9, the histograms of four current states under light illumination and in the dark both exhibit a single exponential behavior.
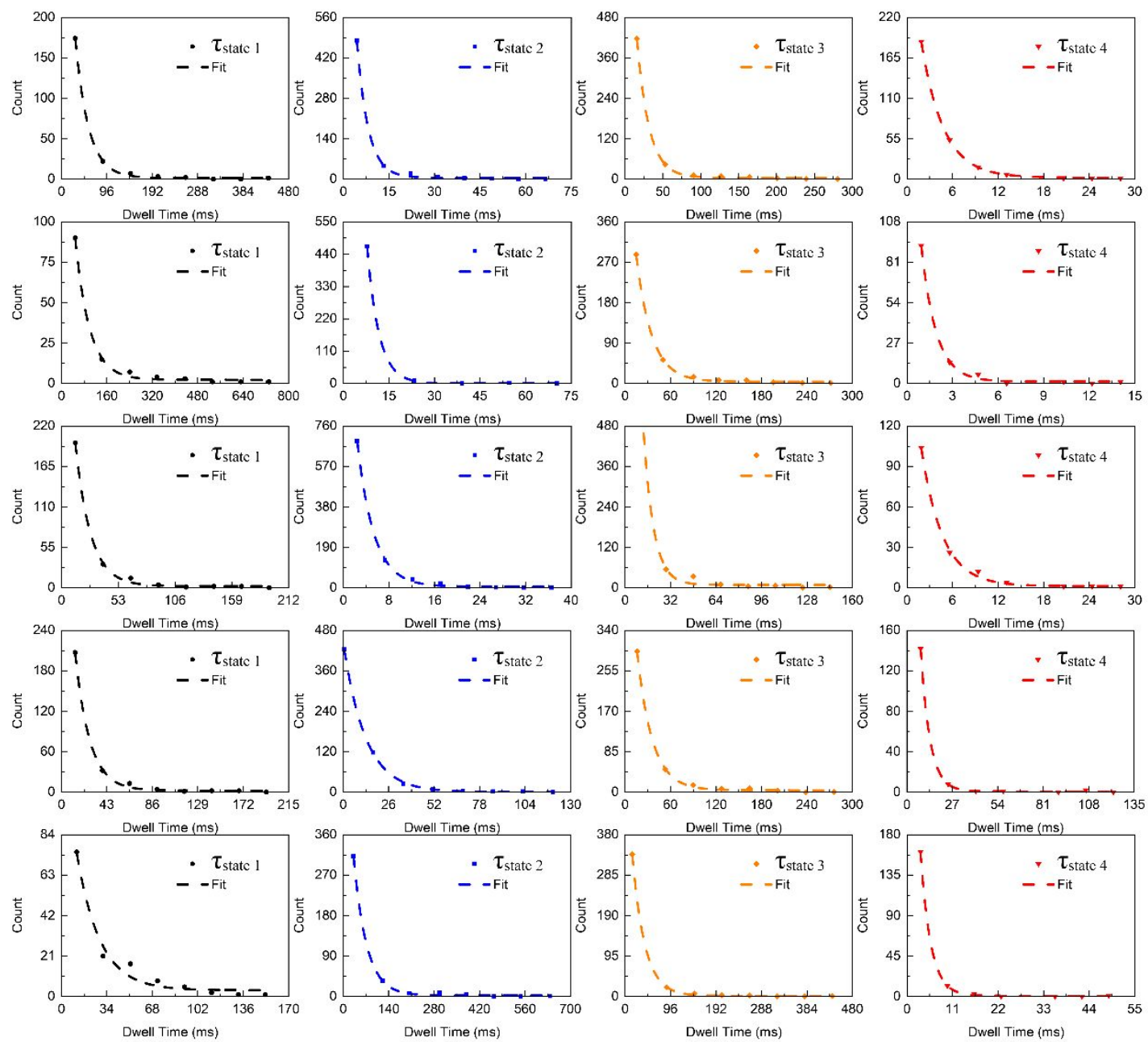

Figure S8. Statistical analysis of LH1-RC temperature-dependent experiments in the dark. These figures show the dwell-time distributions for State 1, State 2, State3 and State 4 corresponding to a series of temperatures from $15^{\circ} \mathrm{C}, 25^{\circ} \mathrm{C}, 35^{\circ} \mathrm{C}, 45^{\circ} \mathrm{C}$ to $55^{\circ} \mathrm{C}$ in the dark. 

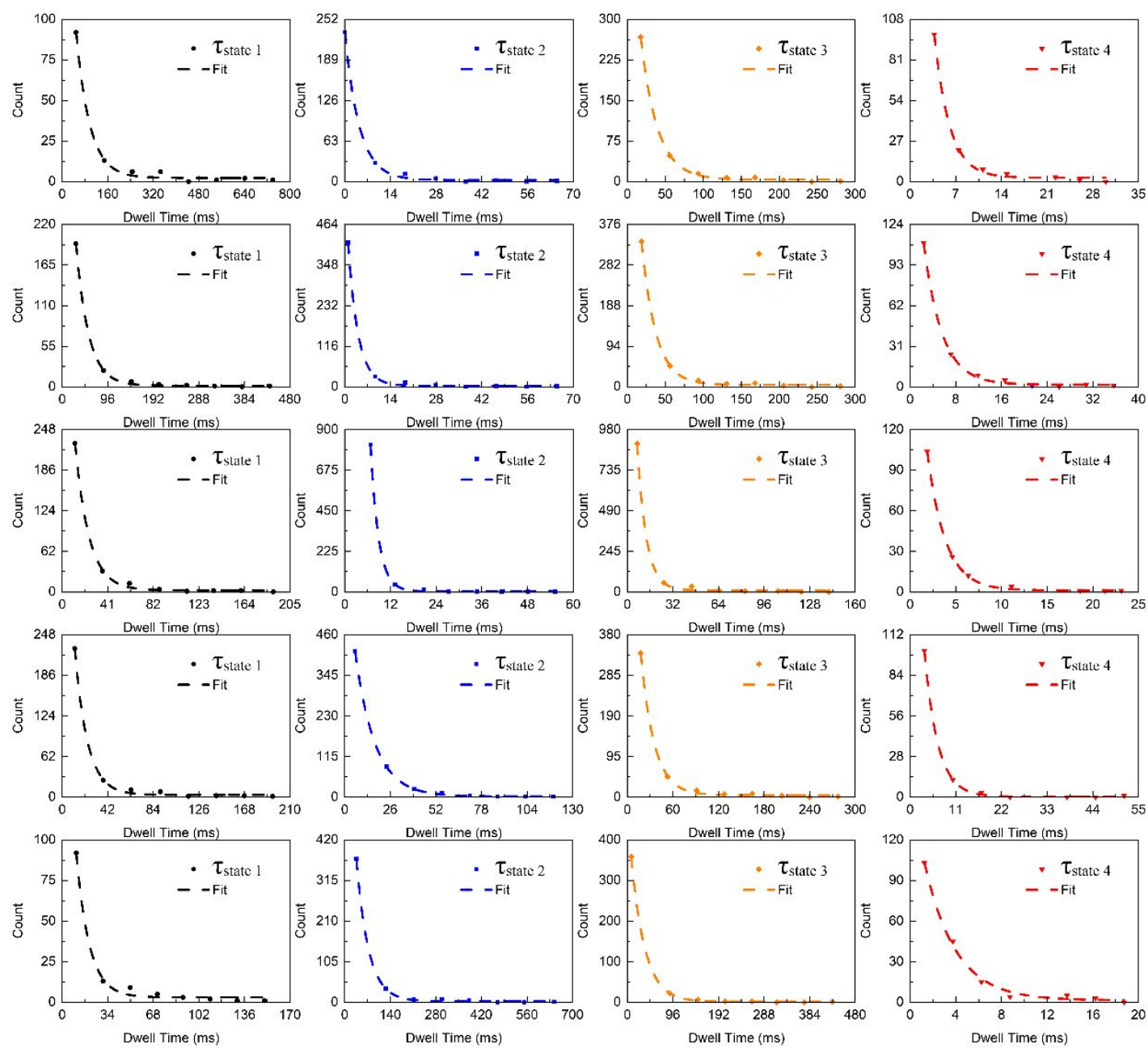

Figure S9. Statistical analysis of LH1-RC temperature-dependent experiments under light illumination. These figures show the dwell-time distributions for State 1, State 2, State 3 and State 4 corresponding to a series of temperatures from $15^{\circ} \mathrm{C}, 25^{\circ} \mathrm{C}, 35^{\circ} \mathrm{C}$, $45^{\circ} \mathrm{C}$ to $55^{\circ} \mathrm{C}$ under light illumination. 
Table S1. The dwell times of four states among different temperatures.

\begin{tabular}{cccccc}
\hline \multirow{2}{*}{$T$} & State & 1 & 2 & 3 & 4 \\
& Parameter & $\tau(\mathrm{ms})$ & $\tau(\mathrm{ms})$ & $\tau(\mathrm{ms})$ & $\tau(\mathrm{ms})$ \\
\hline \multirow{2}{*}{$15^{\circ} \mathrm{C}$} & Dark & $56.05 \pm 1.17$ & $3.82 \pm 0.22$ & $17.04 \pm 0.06$ & $1.77 \pm 0.09$ \\
& Light & $48.89 \pm 1.13$ & $4.42 \pm 0.28$ & $19.38 \pm 0.23$ & $0.99 \pm 0.11$ \\
$25^{\circ} \mathrm{C}$ & Dark & $48.67 \pm 0.87$ & $5.14 \pm 0.13$ & $15.77 \pm 0.22$ & $1.75 \pm 0.09$ \\
& Light & $29.86 \pm 0.87$ & $5.35 \pm 0.09$ & $16.05 \pm 0.21$ & $1.36 \pm 0.14$ \\
$35^{\circ} \mathrm{C}$ & Dark & $14.99 \pm 0.79$ & $6.16 \pm 0.11$ & $10.26 \pm 0.10$ & $1.22 \pm 0.17$ \\
& Light & $14.35 \pm 0.68$ & $6.65 \pm 0.09$ & $10.64 \pm 0.07$ & $1.09 \pm 0.08$ \\
$45^{\circ} \mathrm{C}$ & Dark & $15.35 \pm 1.64$ & $11.76 \pm 0.26$ & $16.57 \pm 0.42$ & $0.97 \pm 0.21$ \\
& Light & $14.88 \pm 0.57$ & $10.13 \pm 0.07$ & $16.75 \pm 0.33$ & $0.94 \pm 0.11$ \\
\multirow{2}{*}{$55^{\circ} \mathrm{C}$} & Dark & $12.99 \pm 1.52$ & $38.07 \pm 0.06$ & $28.34 \pm 0.67$ & $0.95 \pm 0.09$ \\
& Light & $11.95 \pm 1.60$ & $36.68 \pm 0.13$ & $29.74 \pm 0.10$ & $1.07 \pm 0.19$ \\
\hline
\end{tabular}




\section{References}

(1) Patolsky, F.; Zheng, G. F.; Lieber, C. M. Fabrication of Silicon Nanowire Devices for Ultrasensitive, Label-free, Real-time Detection of Biological and Chemical Species. Nat. Protoc. 2006, 1, 1711-1724.

(2) He, G.; Li, J.; Ci, H. N.; Qi, C. M.; Guo, X. F. Direct Measurement of Singlemolecule DNA Hybridization Dynamics with Single-base Resolution. Angew. Chem. Int. Ed. 2016, 55, 9036-9040.

(3) Zheng, G. F.; Gao, X. P. A.; Lieber, C. M. Frequency Domain Detection of Biomolecules using Silicon Nanowire Biosensors. Nano Lett. 2010, 10, 3179-3183.

(4) Yang, C.; Liu, Z. T.; Li, Y. W.; Zhou, S. Y.; Lu, C. X.; Guo, Y. L.; Ramirez, M.; Zhang, Q. Z.; Li, Y.; Liu, Z. R.; et al. Electric Field-catalyzed Single-molecule DielsAlder Reaction Dynamics. Sci. Adv. 2021, 7, eabf0689. 